\title{
An Investigational Analysis of different Approaches and Techniques for Time Synchronization in Wireless Sensor Network
}

\author{
K. Nagarathna \\ Research Scholar \\ Visvesvaraya Technological University \\ Belgaum, Karnataka, India
}

\author{
Jayashree D.Mallapur \\ Professor \\ Dept. of Electronics \& Communication Engg. \\ Basaveshwar Engineering College \\ Bagalkot, Karnataka, India
}

\begin{abstract}
Time synchronization is one of the important research aspect from past decade due to its close correlation on quality of service (QoS) attributes in wireless sensor network (WSN). One of the challenging issues is addressing time synchronization is that different WSN applications have particular QoS demands that varies from throughout, delay, channel capacity, and protocol efficiency. In order to achieve maximized QoS parameters in optimal use of energy various soft computing approaches towards coverage, connectivity, routing, aggregation and fusion etc. are in the main focus of the researchers, whereas in a real life scenario all the soft computing approaches faces serious discrepancies in performance as these protocol do not suggest and correlate the issues related to the time synchronization challenges in wireless sensor network. This paper discusses every technical aspect from fundamental level of challenges in time synchronization in WSN, its type and approaches of time synchronization in general to specific to the wireless sensor network. It also highlights the open issues and future directions of the research. This paper will be one-handed reference article for the academician and researchers for time Synchronization issues and techniques.
\end{abstract}

\section{Keywords}

Quality of Service, Time Synchronization, Wireless Sensor Network.

\section{INTRODUCTION}

The area of wireless sensor network is one of the most addressed and adopted research topic from last decade owing to its advantageous features as well as inherited issues associated with it. The wireless sensor network comprises of sensor nodes which are distributed or positioned in the area where the investigator wants to explore / analyse the data. Such sensor nodes are usually a hardware component which can perform the sensing of the physical data e.g. temperature, thermal, pressure, moisture, smoke, motion, etc. Usually sensors are deployed on the area where it is quite difficult for human to reach and perform investigation on the data of interest. Hence, the data being captured by the sensor nodes are of highly valuable in nature and various internal and external factors affects the effectiveness of the outcome. The external factor being the climatic condition, interference of other RF based hardware device, etc. whereas the internal factor being energy, routing, quality of services, security, etc. From the last decade, there are enough studies being incorporated to mitigate these issues, out of which time synchronization issues [1] is still an unsolved area of research in wireless sensor network. Every sensor nodes in wireless sensor network consists of hardware based local clock, which keeps a track of time for event being captured as well as event being processed by the node. The data packet captured by the sensor node is eventually being identified with the assistance of the value of time-stamped by the sender sensor node. Therefore, as in large scale wireless sensor network, there are high number of availability of sensor nodes, hence, fluctuation of local time (called as clock drift or offset by some studies) negatively affects the wireless sensor network. Therefore, it is essential that issues of Time Synchronization in wireless area network be investigated properly as there are various underlying operations carried out the sensor nodes that are directly dependent on the time synchronization phenomenon. In case of absence of common time factor among the sensor nodes, the time estimation will be redundant, which gives rise to all false positives in event generation and processing method by the sensor nodes. Studies like [2][3] also exhibited that time synchronization could be also used in optimizing the network lifetime of wireless sensor network.

Therefore, time synchronization is an essential operation being carried out in the wireless sensor network application, whose accuracy limit should be as high as possible exclusively in time critical applications like environmental calamities [4], military operation [5], seismic variable observation [6] etc. The prime attempt of this manuscript is to discuss the existing techniques of time synchronization techniques from the macro and micro analysis viewpoint so that the outcome of the study really do assist the future researcher to understand the level of contribution being proposed by various researchers as well as amount of complexities being associated with the evolution of novel techniques. Section 2 discusses about the background of the proposed paper highlighting the significance of time synchronization followed by brief discussion of various existing research contribution in section 3. Section 4 discusses about the standard techniques of time synchronization followed by Section 5 that discusses about the soft computing approaches in the area. Section 6 discusses about the research challenges being explored from the existing literatures. Finally Section 7 makes some concluding remarks.

\section{TIME SYNCHRONIZATION IN WSN}

With the recent advancement in the area of time synchronization in wireless sensor network, there exists various a theoretical studies [7] that discusses about the evolution of low-powered sensor nodes as well as actuators. Time Synchronization is an effective operation highly 
required for distributed wireless sensor network. Some of the critical application in distributed wireless sensor network are like measuring the velocity of a node [8], performing localization based on acoustic signals [9], beam forming operations in distributed manner [10] etc. Unfortunately, the significance of time synchronization is very different in ordinary networking system to wireless sensor networking system. One of the significant factors of the wireless sensor network is the energy depletion, which are very important aspect for every meaningful computational viewpoint. Another significant factor is that various types of applications in wireless sensor network demands all different needs of time synchronization techniques. No single or standard time synchronization technique is applicable in all the applications in wireless sensor network. It was also seen that time synchronization plays a crucial role in data aggregation in wireless sensor network [11]. Right from capturing the data by the member node, forwarding the data to the cluster node, and then forwarding the aggregated data to the base station (or sink), Time Synchronization plays a very critical role in every stages of data aggregation [12]. Time synchronization also plays a critical role in data aggregation by removing the redundant data packet during capturing and processing of the data packet from the other cluster nodes present in the simulation area. For the purpose of sensor node to perform synchronization, the sensor nodes will have to perform communication within certain duration of time and thereby compute an effective estimation of delay [13]. Another significant factor affecting the performance is the asymmetric delay caused by round trip delivery of message [14] and thereby yielding to average errors in computing time synchronization. From micro-analysis viewpoint, the delay estimation, exclusively in WSN, is governed by 4 parameters, which are briefly discussed as below:

\subsection{Source Node Active Time (SoNAT)}

SoNAT can be defined as the actual time consumed by the transmitting node for the purpose of constructing a message that usually consists of processing kernel protocol as well as various types of variable delay caused by the operating system [2]. Moreover time required for the communication of the message from the transmitting node to its network interface further aggravate the issue. The work done by Sichitiu and Veerarittiphan [15] have addressed this issue and presented a soft computing approach of enhancing time synchronization at the time of performing data fusion.

\subsection{Channel Scheduling Time (CST)}

CST can be defined as the estimated delay owing to the waiting for the accessing the transmission channel as the access time is highly MAC (Medium Access Control) dependent. The contention based techniques usually wait for the communication channel to be cleared before even performing transmission or even re-transmission for the situation of collision of packets. It was also seen that majority of the RTS/CTS policies that exists in standard 802.11 based networks will require an exchange of the control packets before even performing the data transmission [2]. Studies performed by Geylan et al. [16] have addressed such issues by introducing a framework that neglects the information pertaining to forwarding time and access time and minimize the time synchronization error drastically.

\subsection{Transmission Time (TT)}

TT can be defined as the time period for the control message to travel to receiver node from the transmitter node using a random selection of channel by the transmitter. For the situation when the transmitting and the receiving node share the similar physical information, such time (TT) is found to be quite minimal. Moreover, the delay factor is substantially governed in distributed wireless sensor network by the TT value as it usually consist of computing the delay caused due to queuing mechanism adopted as well as delay owing to switching mechanism on each router while the message propagates through the network. The study performed by Tanvir et al. [17] have discussed such issues and have proposed a solution based on transmission characteristics of the routing beacons for the purpose of addressing the localization problems and therefore mitigating the errors in time synchronization in wireless sensor network.

\subsection{Sink Node Active Time (SiNAT)}

SiNAT can be defined as the time for the network interface to receive the control message by the receiving node and simultaneously update the transmitting node after its arrival [2]. If the arrival time is found to be time-stamped minimally, than the receiving time will not maintain the overhead of the context switches, system calls, or even message transferred information. Studies performed by Zhang and Deng [18] have discussed such aspects where the authors have used the parameters from the receiving time and performed the time synchronization probabilistically in wireless sensor network.

The time synchronization techniques in the area of the wireless sensor network basically have emerged out with techniques for providing an effective synchronization of the hardware based local clocks embedded in sensor nodes [19]. Time synchronization also permits the group based communication with higher degree of supportability on cooperation among the sensor nodes. The synchronization technique also assists in supporting operations like data fusion, data aggregation, coordinating with sleep and wake cycles among the nodes, identifying a significant event etc. Every sensor nodes operates on its own local clock and it receives or sends the data packet from / to other sensor nodes at a sampling rate based on its own local clock. It was also seen that sensor nodes switches over to sleep mode for the purpose of preserving the power. Hence, if the clocks in the sensor nodes are not effectively synchronized, the sensor nodes will miss the data packets being transferred by the other sensor nodes. Therefore, it is extremely important that local clocks to be properly synchronized so that the global clock have less probability of shooting significant average synchronization errors [20]. The literature was also found with three fundamental synchronization approaches as follows:

\subsection{Relative Ordering}

Relative ordering is the technique performed by the sensor node that maintains the order to control messages or captured events in the process of performing time synchronization. Study carried out by Shtossel et al. [21] was found to use the technique of relative ordering for designing habitat monitoring application in wireless sensor network.

\subsection{Relative Timing}

Relative timing is a method adopted by the sensor node by maintaining its clock drift and clock offset related information with the neighboring nodes in wireless sensor network. Hence, a better synchronization is possible with one sensor node with its neighboring sensor nodes. Studies conducted by Simon [22] have used relative timing using efficient ring topology for enhancing time-synchronization in wireless sensor network. 


\subsection{Global Synchronization}

Global synchronization is a process of performing synchronization considering all the local clocks available to be synchronized with the formulated global clock. Such method gives better usability and better synchronicity in the distributed wireless sensor network. The work discussed by Yoon and M.L. Sichitiu [23] have discussed using global synchronization technique.

Therefore, it can be seen that there are massive set of requirements against time synchronization issue, such requirements should be studied effectively as the performance metric too. Hence, various issues associated with the time synchronization with respect to the existing system are as follows:

\subsection{Energy Efficiency}

An effective time synchronization algorithm also calls for energy efficiency requirements as the sensors are basically resource constraint. Consideration of energy efficiency in time synchronization can be seen in the study of [24] and [25].

\subsection{Scalability}

An effective time synchronization scheme calls for meeting the better scalability requirements of the sensor network. A better time synchronization scheme should also support increasing number of node density. Consideration of scalability is seen in the study of [26] and [27].

\subsection{Precision}

Precision is completely dependent on various types of application and hence to uniform rule can be cited in this case. However, an effective time synchronization scheme should also ensure better precision level to show its effectiveness in reducing synchronization error with entire network. The author of [28] has considered precision factor in his study for enhancing issues of time synchronization.

\subsection{Robustness}

The wireless sensor network is highly prone for node to malfunction due to various security, technical, as well as environmental reasons. Hence, a better time synchronization technique should be highly robust in its operation even when some sensor nodes are found to be not working properly. The author of [29] has considered security and robustness for performing loose time synchronization in WSN.

\subsection{Lifetime}

A better time synchronization scheme should also ensure better network lifetime to ensure its effectiveness. The author of [30] discussed about lifetime attribute as the potential factor of time synchronization.

\subsection{Scope}

An effective synchronization scheme should have better global time based for all the sensor nodes in the cumulative network. Owing to the issues pertaining to scalability, global synchronization is highly challenging to accomplish in large scale wireless sensor network. Hence, an effective time synchronization scheme should have higher scope for global synchronization [30] and better supportability of the large scale wireless sensor network.

\subsection{Cost and Size}

Conventional theory as well as evidences from literature eventually discusses that sensor nodes are highly resource constraint and expensive in nature owing to the hardware based implementation. Hence, any novel discussion of time synchronization technique should ensure cost effectiveness and size of network. Discussion of cost and size in viewpoint of time synchronization was seen in the study of Kim et al. [31].

\subsection{Immediacy}

Some of the applications of the sensor network are based on time bound as well as mission critical requirements (e.g. intruder identification, chemical plant, gas leak identification etc.). Hence, any new discussion of time synchronization technique should ensure that they can effectively manage and perform time-bound and mission critical application with highly reduced error. The issue of immediacy is discussed by Cheng [32] in their study.

\section{RESEARCH CONTRIBUTION}

The discussion on the time synchronization issues in wireless sensor network furnishes a better scope of performing indepth investigation. Synchronization plays a critical role in wireless sensor network as it calls for cooperative approach from all the sensor nodes as well as sophisticated task of the data transmission [33][34]. Owing to the significant contribution of the researchers of the past decade, various computational as well as analytical techniques have been evolved. Such techniques were found to be frequently adopted even in ongoing research activities. The usability of the sensor network calls for catering up the requirement of the user queries by aggregating the data from each sensor. In order to accomplish such objective, it is very important that there are extensible considerations based on in-depth mathematical investigation. Hence, various schemes, evaluation techniques, and analysis approach that are used in the existing studies are discussed in this section as follows:

Master-slave versus peer-to-peer synchronization

\subsection{Master-Slave}

Such type of the technique allocates a one sensor node as masters while other sensor node as slave [35]. The protocol attempts to read the local clock as the time reference and perform time synchronization with the master. Good example of master slave protocol is TSPN or FTSP. There is a higher consumption of CPU resources by the master sensor node as compared to the slave sensor nodes and therefore only the sensor nodes with better resource availability is selected as master sensor node.

\subsection{Peer-to-Peer}

Such type of the protocol applies peer-to-peer technique for accomplishing the communication among the sensor nodes. Good example for this protocol could be RBS and TDP [35]. The peer-to-peer protocol also relieves the threat of master sensor node failure and thereby offers better flexibility.

Probabilistic/Deterministic synchronization

\subsection{Probabilistic Synchronization}

In this synchronization process, the value of the clock offset is evaluated using probability theory as deterministic theory usually bounds the system to perform extended transmission of message transfer and extra usage of computational resources. Deterministic techniques are therefore less supported in time synchronization technique in wireless sensor network [36]. The studies performed by authors in [37] have analyzed event interaction using this scheme on parallel simulation. 


\subsection{Deterministic Synchronization}

Adoption of deterministic technique only ensures the maximal limit of the clock offset with certainty. The frequently adopted algorithms like RBS and TDP are basically deterministic in nature. The author of [38] has discussed about the use of deterministic clock synchronization performed in TinyOS. Clock Correction untethered clocks.

\subsection{Clock Correction}

During time synchronization process, the function of the clock memory will be required to be updated. Adoption of clock correction technique is seen in TSPN protocol [6]. Moreover, the authors of [39] have developed a new time synchronization protocol to perform drift compensation mainly.

\subsection{Untethered Clocks}

Majority of the clocks in the sensor nodes are autonomous in nature but each sensor node records the data required to convert the local time into the time base of each other. The author of [40] has used a one-way untethered clock approach for designing efficient time synchronization for WSN. The system is found to be appropriate used in wireless networking environment.

Internal synchronization versus external synchronization.

\subsection{Internal Synchronization}

The objective is to minimize the maximum difference between the readings of the clocks of the sensors. Can be performed in master slave or peer to peer [6].

\subsection{External Synchronization}

A source of standard time such as universal time is provided here. We do not need a global time base since we have an atomic clock that provides real-time in the real world usually called the reference time to be synchronized. Cannot be done with Peer to Peer [6].

The use of internal and external synchronization was found in the recent study of [41] who has used a fault tolerant policies for performing efficient time synchronization during data dissemination process in WSN.

\section{Clock Overheads in Communication.}

\subsection{Sender-to-Receiver}

Most existing methods are transmitter to a receiver by transmitting the values of the clocks. In study of [42], poor drift estimations was done using sender-to-receiver synchronization policy. Therefore these methods are faced with variances of the delay message. The transmitting node periodically sends a message with its local time as a timestamp to the receiver and then the receiver synchronizes with the sender using the timestamp receiver from the sender.

- The transmitting node sends periodically a message with its local time as a timestamp by the receiver.

$\circ \quad$ The receiver then synchronizes with the sender using the times tamp received from the sender.

- The delay message between sender and receiver is calculated by measuring the total time to and from the moment the receiver requests Timestamp juiceonly time he receives an actual response

\subsection{Receiver-to-Receiver}

This is one of the approaches for performing time synchronization in WSN. This approach uses the property of the physical broadcast medium that if any two receivers receive the same message in a single-hop transmission in WSN, they receive it at approximately the same time. The authors of [43] have used this type of receiver-receiver synchronization method to evaluate overhead and error in WSN. Each sensor node has a local clock. In this clock a quartz crystal oscillator is used. Frequency of quartz crystal oscillator changes with change in ambient conditions, hence local clocks run faster or slower as compared to global clock (real time). The author has mathematically represented it by considering $h(t)$ to be reading of local clock of node at real time t. Hence, the rate $\alpha(\mathrm{t})$ at which the nodes clock runs is mathematically represented as [44],

$$
\alpha(t)=\frac{d h(t)}{d t}
$$

Then, evaluation of the local time of any sensor node is,

$$
H(t)=\alpha(t) \cdot t+\beta(t)
$$

Where $\beta(t)$ is the estimated offset of the sensor node. The variable clock offset is basically computed as the difference between the local and global clock. In the above eq.(2), the clock rate $\alpha(t)=d h(t) / d t$ posses a value equal to unity for a precise clock computation at all times. However, such clock is anticipated not to run in either very fast or very slow and only intends to display the correct time only. However, it should be known that the frequency of the quartz oscillator of the sensor node's clock eventually changes with any slight alteration in the ambient temperature, humidity, or supply voltage. Applying probability theory, the rate computed for local clock to run either faster or slower is only 1 . Hence, the clock drift of a sensor node is represented as standard deviation of the rate of clock from value of unity. Hence, clock drift for any sensor node can be mathematically represented as,

$$
\rho(t)=\alpha(t)-1
$$

As, the system applies probability theory, so clock drift $\rho(t)$ are restricted to the range of 0 and 1 . And therefore, $1-\rho(t) \leq$ $\alpha(\mathrm{t}) \leq 1+\rho(\mathrm{t})$.

Unfortunately, clock drift of the sensor node is not that easy to mitigate effectively as there are various external factors like pressure, temperature, supply voltage, temperature, as well as environmental alteration. Some of the other reasons are i) initiation of nodes at different time, ii) frequency difference for the clock oscillation resulting in skew, iii) variable frequency over time owing to energy depletion resulting in drift. Although, evaluation of clock drift has been investigated in operating system, but with the presence of tiny operating system (TinyOS) in wireless sensor network, an additional complexity arises owing to inherent characteristics of the sensor nodes.

\section{STANDARD TECHNIQUES OF TS}

This section will discuss the standard techniques of time synchronization that have been evolved from the continuous effort from the international research communities. Although the concept of time synchronization was actually pioneered by Elson and Estrin [45], but various other techniques have also emerged out, which were seen very often to be either referred or studied by various researchers in the recent past. Some of the standard approaches explored are:

\subsection{Reference Broadcast Synchronization (RBS)}

RBS is one of the significant attempted techniques introduced by Elson et al. [45] that uses physical layer broadcast for the purpose of comparing the local clocks from receiver node viewpoint. The potential highlight of RBS scheme is that the receiving sensor nodes broadcast the reference beacons to 
their adjacent sensor nodes which arrival time instead of using timestamp. Interestingly, the sensor node just broadcast a unit pulse to two receiving sensor nodes, which after receiving, performs exchanging of their receiving time of pulse and attempt to evaluate their relative phase offsets. RBS scheme was also extended to incorporate certain features like permitting the time synchronization operation to be carried out by two receivers and enhancing the quantity of the reference pulse to accomplish improved precision in synchronization of local to global clocks.

\subsection{Time Synchronization Protocol for Sensor Networks (TSPN)}

TSPN is another frequently adopted technique to perform time synchronization, exclusively for the entire network scenario. TSPN initially establishes hierarchical structure of the network and then perform pair-wise synchronization along the edges of this structure for accomplishing better global clock synchronization of the entire network. After this step, all the sensor nodes then perform their clock synchronization with the reference sensor node. This phase of operation is carried out using level discovery stage and synchronization stage. The motive of level discovery phase is to generate a hierarchical topology in the wireless sensor network by assigning a level to each sensor node. It considers root node as a sensor node with 0 assigned level and therefore, $i^{\text {th }}$ level of one sensor node is synchronized with $\left(\mathrm{i}^{\text {th }}-1\right)$ level of another sensor node. Finally in the end stage (or level), it was seen that all the sensor nodes are actually synchronized to the root node and therefore the entire network nodes accomplishes their effective synchronization. The study of TSPN was done using real-time Berkley's Mica architecture and also deploys time stamping the data packets at MAC layer for the purpose of removing uncertainty of synchronization in transmitting node.

\subsection{Tiny sync and Minisync (TS/MS)}

TS/MS is another time synchronization technique that uses concept of pair-wise synchronization [46] using multiple levels of round-trip evaluation. TS/MS also adopts line-fitting mechanism to accomplish the offset as well as differences in rate of two sensor nodes. TS/MS uses constant-rate framework where the data points are obtained using multiple level of round-trip synchronization process. The TS/MS technique adopts the simple mechanism of evaluating the relative offset and clock-drift of two nodes. The $1^{\text {st }}$ sensor node time-stamps and forwards e probe control message to the $2^{\text {nd }}$ sensor node. A time stamp is generated by the $2^{\text {nd }}$ sensor node after getting the control message and instantly forwards a reply message to the $1^{\text {st }}$ sensor node. Final timestamping is done by the $1^{\text {st }}$ sensor node after receiving the reply from the $2^{\text {nd }}$ sensor node. The backbone of the TS/MS operation is basically the set of data points which ensures the improvement of algorithm precision with the increase of data points. The principle of formulation of the data points is based on constraints pertaining to relative offset and drift. Mini-sync is the advanced version of the Tiny-Sync for exploring the better solution with enhanced complexities. The system also prevents TS/MS for truncating the consideration of constraints for future data points.

\subsection{Lightweight Tree-based Synchronization (LTS)}

Different from other time synchronization technique, LTS was developed for reducing the complexities associated with time synchronization technique and not for enhancing the accuracy or precision. Hence, LTS considers accuracy as the constraint and performs computation to resolve the complexities associated with it. LTS system ensures that the time accuracy is very less, which gives a room to use lightweight time synchronization algorithms in wireless sensor networks. Literature have witnessed LTS scheme to work on pair-wise synchronization scheme where the techniques calls for sensor node to perform synchronization to a specific reference point (e.g. base station). The technique usually adopts centralized scheme and uses spanning tree approach followed by formulation of pairwise synchronization along the $(n-1)$ edges of the spanning tree. The root is considered to be always a reference sensor node of the spanning tree to perform an additional operation of resynchronization, if required. Considering bounded clock drifts and anticipated precision, the root estimates the time duration for valid unit step of synchronization. The system of LTS also ensures that depth of the tree do not have a significant effect on the time to perform synchronization of the entire network along with adverse effect of error among the leaf nodes. The information pertaining to the depth of the tree is traversed back to the root for the purpose of performing resynchronization. The LTS technique also supports multihop communication in highly distributed manner. It also chooses not to use spanning tree sometimes when the sensor node choose to perform its own synchronization. In such cases, the sensor nodes transmit the synchronization request to the nearest root, which ensures the synchronization with root node to all the sensor nodes leading to the root node. The potential advantage of such policy is ensuring participation of all the node information however insignificant it may be. Also, as the sensor nodes perform their own synchronization, hence unwanted computational effort of performing synchronization is highly reduced. However, LTS technique may aggravate the number of pairwise synchronization as the node decides on their own resulting in unwanted usage of available resources in wireless sensor network. Such issues have been addressed in the work of Holczer [64] and Song et al. [65].

\subsection{Time Diffusion Synchronization (TDP)}

TDP scheme has higher supportability of the entire network. In this scheme, a group of master sensor nodes are selected that should have a proper access to global time for performing global synchronization. A request message consisting of current time, control message of receiver is broadcasted by the master node, which the computes the mean delay as well as standard deviation. On the other hand, the receiving sensor nodes perform and iterative process of aggregating such information for using by them for marking themselves as diffused leaders. The mean delay estimates as well as standard deviation are added along the route from the master sensor nodes. The schemes of TDP have some of the wide range of sub-techniques for autonomously performing synchronization of the nodes and reducing the false alarm on the other hand. The TDP scheme also performs a better balance between the time synchronization as well as delay estimation and was also found to be quite favorable to distributed wireless sensor network. However, at the initial level, the sensor nodes may receive a preliminary pulse from the base station either using multi-hop flooding or using direct broadcasting. Such sensor nodes deliberately seek to call themselves master node with the process of election of either master or diffused leader node. The technique usually intends to perform reduction of false positives and effectively balance the load of the network. The system also performs peer-evaluation technique for minimizing the false alarm from becoming the master node or diffused leader sensor node. Some of the effective benefits of 
adopting TDP scheme as discussed by Gu et al. [47] are as follows: i) TDP is highly fault tolerant against message losses, ii) TDP supports better equilibrium of load in the network, iii) TDP also supports mobility actor in wireless sensor network, and iv) TDP is highly flexible as well as fault tolerant. However, the author have also chalked out some of the demerits of the TDP as i) higher convergence time and ii) backward running of clock. The higher convergence time was observed when the system doesn't consider external precise time servers and clock runs backward when the clock value was found to be unexpectedly adjusted to the lower value. Obviously, these are the cases of inefficient and unreliable time synchronization process.

\subsection{Asynchronous Diffusion (AD)}

$\mathrm{AD}$ has the higher level of supportability of internal synchronization (TDP scheme supports external synchronization) [48]. In $\mathrm{AD}$, each sensor node occasionally transmits a broadcasted message to its adjacent sensor nodes that replies with the message consisting of their current time owing to time stamping method. The receiving sensor nodes then evaluates the mean of the time-stamp being received and then broadcast the mean estimates to the adjacent sensor nodes for updating the time scale.

Table 1 Performance Comparison

\begin{tabular}{|l|l|l|l|l|}
\hline $\begin{array}{l}\text { Subjective } \\
\text { Performance }\end{array}$ & Accuracy & $\begin{array}{l}\text { Energy } \\
\text { Conservation }\end{array}$ & Scalability & Fault Tolerance \\
\hline RBS [45] & Maximum & Maximum & Good & No \\
\hline TPSN [49] & Maximum & Average & Good & Yes \\
\hline TS/MS [50] & Maximum & Maximum & N/A & Yes \\
\hline TDP [51] & Maximum & Average & Good & Yes \\
\hline AD [48] & Unidentified & Low & N/A & Yes \\
\hline
\end{tabular}

\begin{tabular}{|l|l|l|l|}
\hline $\begin{array}{l}\text { Measurable } \\
\text { Performance }\end{array}$ & Precision & Convergence time & Network Size \\
\hline RBS [45] & $1.85 \pm 1.28 \mu \mathrm{s}$ & N/A & $2-20$ Nodes \\
\hline TPSN [49] & $16.9 \mu \mathrm{s}$ & Unidentified & $150-300$ Nodes \\
\hline TS/MS [50] & $945 \mu \mathrm{s}$ & Maximum (Multihop) & N/A \\
\hline TDP [51] & $100 \mu \mathrm{s}$ & Maximum & 200 Nodes \\
\hline AD [48] & Unidentified & (Multihop) & $200-400$ Nodes \\
\hline
\end{tabular}

The effectiveness of the standard time synchronization techniques are usually evaluated using two types of performance parameters. The first type of performance evaluation parameters is called as subjective performance is evaluated using accuracy, energy conservation, scalability, and fault tolerance. The second type of performance evaluation parameter is called as measurable parameters which are usually evaluated using precision, convergence time, and network size. Table 1 highlights some of the significant statistics of subjective as well as measurable performance parameters for evaluating time synchronization in wireless sensor network. Precision is one of the important evaluation parameters in this process that computes the highest possible error in forms of offsets and skews for the node's local clock with respect to global clock. However, accuracy, scalability, fault tolerance are other subjective parameters which are also given equal importance. Energy conservation basically highlights the effect of timesynchronization on the energy-constraint sensor nodes in wireless sensor network. From mathematical viewpoint, convergence time as well as precision is found to be another set of measureable performance parameters. Network size is another important parameter using to understand the scalability extent of the algorithm. However, network size should be also discussed from cluster viewpoint also to understand how the time synchronicity can be affected by the network size. However, it was never seen much, if there is a possible of inclusion of other significant parameters too for significantly evaluating time synchronization effectiveness. Table 1 just highlights the frequently used performance evaluation parameters in the existing research work, however, we believe that there is a need to extend the study of time synchronization and check for inclusion of other network or system parameters apart from that highlighted in Table 1.

\section{SOFT COMPUTING TECHNIQUES}

From the previous section 4 , it was seen that time synchronization is one of the critical problem in operational viewpoint of WSN. This section will discuss about some of the famous soft computing techniques that were introduced in the past (and even now) for the purpose of energy efficiency as well as resolving the issues of time synchronization.

\subsection{Coverage \& Connectivity}

The significance of time synchronization technique is quite high in ensuring the effectiveness of coverage and connectivity in wireless sensor network. The term coverage is represented as the estimates of effective observation as well as controlling features of the defined physical space in wireless 
sensor network [52]. The authors in [53] have discussed about their coverage protocol that was designed using probability their considering the sensing framework of wireless sensor nodes. The authors have considered that sensor nodes are well time-synchronized at higher level of accuracy. According to the author, a good assumption in higher accuracy in time synchronization only permits the better coverage schemes that can substantially reduce the adverse effect of clock drift. The study towards time synchronization technique on under water sensor network was discussed by the author in [54]. The study has adopted a simple synchronization protocol for mitigating the adverse effect of mobility as well as the propagation delay. A challenge of multi-hop sensor network scheme was discussed by authors in [55] with a motive on reducing the extent of energy depletion among the sensor nodes. However, the study was only focused on ensuring optimal connectivity and coverage area. The outcome showed better energy efficiency as the sensor nodes are programmed to switch over to sleep mode probabilistically. However, the study didn't addressed complexity of time synchronization for large scale wireless sensor network and outcome was also found to be associated with additional need of communication as well as energy depletion too. The similar problem was also researched by the author in [56] where the author presented certain set of conditions for the sensor nodes to switch over to sleep mode and thereby ensure better network connectivity. The work done by authors in [57] have discussed certain technique to use minimum number of sensor node in awake mode and thereby address effectiveness in coverage and connectivity issues in wireless sensor network. Use of flooding time synchronization was found in the work carried out by author in [47] for time synchronization [13] among the sensor nodes and deluge [58] for the purpose of wireless reprogramming. The authors have presented a framework termed as uSense that is claimed to support numerous categories of coverage techniques with simple switching techniques in wireless sensor networks.

\subsection{Routing Protocol}

Routing protocols plays a critical role in ensuring effective and time-bound communication in wireless sensor network. Use of cross-layer technique was witnessed in the study of author in [59] where the work mainly targets the efficiency of the routing protocol for distributing the time information. The prime motive was to use the optimal duty cycling of the sensor nodes. The significance of this study is that the author have integrated time synchronization technique in network later that was ultimately found with higher supportability on flat as well as non-hierarchical network topologies too. The outcome of the technique was found with better mitigation schemes for control overhead on conventional time a synchronization technique that adopts layer based policy. The analysis of the synchronization error using a reactive routing protocol was found in the study of author in [26]. However, the outcome was associated with loopholes owing to the clock skewness. Another notable work in the direction of routing protocol based solution is introduced by Varagnolo et al. [60]. The work was based on real-time filtering over directed staged flooding and unicast path diversity, which is the significant implement also witnessed in the study of [61], where the authors have used time synchronization techniques on mesh protocols. Both the works ([60], [61]) have associated issues of scalability, which was never found to be evaluated with respect to skew compensation. This is a significant open issues as the errors evolving from skew measurement as well as estimation for skewness errors are anticipated to be propagated in the multiplicative pattern, where the skewness precision and probabilistic higher limit on the error estimation needs to be controlled. The authors have mitigated this problem by configuring a precise minimal and maximal limit on the differences of time of receiving the two control message used for evaluating the skewness. The work has also selected the size of higher dimensional neighboring routing table for computing skewness error. However, the outcome of such idea was never benchmarked properly with respect to its influence in time synchronicity resulting in unsolved issues of energy efficient time synchronous techniques in large scale wireless sensor network.

\subsection{Aggregation}

Time synchronization plays an extremely critical role in data aggregation technique of wireless sensor network. Adoption of time synchronization technique was seen in the work of [62], where the author chooses to perform security on the data being collected by mobile nodes. The work have discussed a new parameter of mobile node that should be authenticated by the leader node using cryptographic key management technique. After successful authentication, the discussed security framework believes that all the sensor nodes are then time synchronized in all the existing clusters, which is something new in cluster based wireless sensor network. Interestingly, the author in [62] have used time synchronization factor as a medium to circumvent the external attacks in wireless sensor network. The study carried out by author in [63] have discussed an aggregation scheme of wireless sensor network considering time constraint for adjusting the aggregation time of the sensor node for the purpose of accomplishing better performance. The study performed by [64] has discussed a data aggregation scheme for performing in-network secure data aggregation. Study in same direction was performed by author in [65] who have used explicitly time synchronization technique as a media to mitigate the security threats in wireless sensor network. Effect of time on data aggregation operation was studied by the authors in [66] who have developed a time based framework for regular aggregation scheme for accomplishing better energy efficiency with maintaining less data redundancy.

Although, majority of the data aggregation based methods have been introduced in the past, but majority of the study considers that time is slotted properly and only single value of time estimates to be processed at unit time slot. However, the significant considerations of the time synchronization were never found to be part of any experiments being carried out till date in this regards. The work done in [67] discusses about the traffic reduction during data aggregation but it doesn't furnish a complete solution to the critical problem associated with time synchronous in wireless network. It was also seen that in majority of the case, the sensor nodes do critically assume require time to be synchronized from global positioning system. However, such schemes may be well build in future application, but existing solution cannot consider a global time references. All the techniques are found to have higher dependability on the control message and their exchanging operation which are not at all cost effective measures to be recommended.

\section{RESEARCH CHALLENGES}

From the Section 3, 4, and 5, it can be seen that there are massive archival of some of the significant techniques that has been introduced by the researcher in the past decade for the purpose of mitigating time synchronization issues in wireless sensor network. It has been found that different authors have their own uniqueness in their approach, where the outcome of their study was evaluated using their assumption and 
experimental test-bed. Various analytical approaches were found in the time synchronization techniques exclusively for single hop network for the purpose of defining the accuracy features of the discussed synchronization techniques. Unfortunately, in case of multi-hop network, there was no evidence of any analytical model that could be adopted for further extension of future research work. It is already known that standard routing protocols that have higher supportability of multihop in wireless sensor network is very limited and no evidence of time synchronization technique considering multihop has added to a significant research gap. This fact needs to be addressed in the future study direction that emergence of increased error rate in time synchronization is quite high when two sensor nodes attempts to perform synchronization in multihop network. Although, effect of time synchronization of multihop network doesn't have much significant effect in pair-wise error evaluation, but still the scope of evaluation in same is extremely few to find in existing studies. Such research gap calls for performing an extended study as well as problem investigation in the line of both single and multihop synchronization techniques in distributed wireless sensor network. The most frequently adopted RBS scheme was found with a significant issue when the identification or the discovery process of the sensor node to be acting as beacon sender evolved. This is a challenging issue as if there is presence of more number of beacons in a single neighborhood, it may lead to evolution of message redundancy as well as control overhead too. This fact will also lead to maximize the consumption of constrained resource available within the sensor nodes. Moreover, such facts could be possibly more investigated to draw the understanding of association between precision as well as redundancy in the viewpoint of time synchronization. Future research work could draw more assumptions and adopt novel techniques for understand the possibility of various number of beacon sends for accomplishing the anticipated minimal synchronized errors. To sum up, the issues observed in standard RBS scheme are i) requirement of additional time synchronization message for exchanging among the sensor nodes, and ii) limitation of the transmission range (adjacent sensor nodes) in RBS scheme. The other adopted scheme of time synchronization (e.g. TPSN [49], TS/MS [50], TDP [51], and $\mathrm{AD}$ [48]) also encounters some of the significant issues. The evolution of time-stamping jitter owing to delay while forwarding the data packet as well as information pertaining to synchronization error difference (due to clock drift) is still unsolved. Although, such technique have its own advantages on its own assumptions, but it should be known that timestamping jitter is the outcome of the variance of the timeinterval between the incorporated time stamp into the data packet by the transmitting node as well as reading it to the receiver. Although the significant work done by Maroti et al. in [13] has done some of the potential contribution in the area of Time Synchronization by addressing the inaccuracy in source of message time-stamping, but still it is associated with large number of issues. Maroti et al. [13] have carried out the study considering channel access, transmission, reception, propagation, and receive time uncertainty. Unfortunately, the error evolved from the outcome was found to be highly arbitrary in nature and challenging to forecast or reduce especially in large scale wireless sensor network. The approach also doesn't ensure if the clock drift can be effectively minimized as it can vary with the variation of temperature and time. Hence, the future study must focus on compensating the clock drift by introducing an approach for regular updating of the information associated with time factor related to sender and recipient sensor nodes. Moreover, energy minimization factor was another unsolved area as exploring the likelihood to enhancing the energy conservation while ensuring both connectivity and coverage can be termed as optimization issue. Unfortunately, no literatures were evident to consider such time synchronization factor effectively for maximum energy preservation in large wireless sensor network.

Another significant issues explored in the review of literature is that majority of the prior studies have not considered appropriate global time synchronization as it is quite challenging for large scale wireless sensor network. It was also seen that existing studies also don't have any effective consideration of the time asynchrony. Such adoption of the techniques could be duly investigated in the future work. Moreover the studies of time-synchronization pertaining to coverage and connectivity in the area of wireless sensor network [52]-[58] have discussed various types of service enhancement parameters like coverage ratio, delay, lifetime etc, but such parameters are not the sole parameters to testify the effectiveness in time synchronicity in large scale wireless sensor network. Also, such techniques are effective on their own assumption, but fail to cater up the various dynamic design approaches of time synchronization problems in wireless sensor network

\section{CONCLUSION}

The present paper has discussed the significant contribution in the forms of techniques introduced by the researchers in the past. With the availability of limited resources in sensor nodes, performing computational analysis and ensuring effective time synchronization was never an easy task. Although, from the evidence being discussed in this paper about the various standard techniques of performing time synchronization, it was seen that almost all the technique have some loopholes that actually doesn't meet the design goal of the wireless sensor network. The extent of analysis outcome was studied for all the published papers and we came to conclusion that each technique have their own advantages and scope, however, problems of error reduction in synchronization is still an open issues. Moreover, the outcome reviewed from all the prior studies doesn't seem to provide an effective benchmarking for which purpose understanding the best standard of time synchronization was quite a challenging task. Hence, our work for future direction will be towards addressing the research gap explored in this paper and introduce a novel framework with support of highly extensive analysis and benchmarking techniques to generate reduced synchronization errors substantially. We will also like to make some sophisticated assumption to design such model and experiment it to understand all the influence of various significant network and system features on time synchronization error reduction.

\section{REFERENCES}

[1] Ghosh, S.2006. Distributed Systems: An Algorithmic Approach, Chapman and Hall/CRC Press, Computers, 1st Edition,,ISBN-10: 1584885645, pp. 424

[2] Boukerche, A.2006. Handbook of Algorithms for Wireless Networking and Mobile Computing, CRC Press, Computers, ISBN 9781584884651, pp.424

[3] Dargie,W, Poellabauer, C.2010.Fundamentals of Wireless Sensor Networks: Theory and Practice, John Wiley \& Sons, Technology \& Engineering, ISBN: 9780-470-99765-9,pp. 336 
[4] Eliassen, F, Kapitza, R.2010. Distributed Applications and Interoperable Systems, Springer, Computers, ISBN 978-3-540-68639-2, pp. 243

[5] Kitsos,P.2010. Security in RFID and Sensor Networks, CRC Press, - Technology \& Engineering, ISBN-10: 1420068393, pp. 560

[6] Akyildiz,I.F, Vuran, M. C.2010.Wireless Sensor Networks, John Wiley \& Sons, Technology \& Engineering, ISBN: 978-0-470-03601-3, pp. 520

[7] W. Dargie, C. Poellabauer, Fundamentals of Wireless Sensor Networks: Theory and Practice, John Wiley \& Sons, Technology \& Engineering - 336 pages, ISBN: 978-0-470-99765-9, 2010

[8] Cerpa. A., Elson. J., Estrin, D., Girod, L., Hamilton, M., and Zhao, J.2001. Habitat monitoring: Application driver for wireless communications technology, Proceedings of the ACM SIGCOMM Workshop on Data Communications in Latin America and the Caribbean

[9] Girod, L.2000. Development and characterization of an acoustic rangefinder, Technical Report 00-728, University of Southern California, Department of Computer Science

[10] Yao, K., Hudson, R., Reed, C., Chen, D., and Lorenzelli, F.1998.Blind beamforming on a randomly distributed sensor array system, IEEE Journal of Selected Areas in Communications,Vol.16, Iss.8, pp.1555-1567

[11] Pottie, G., Kaiser, W.2000.Wireless integrated network sensors". Communications of the ACM, Vol.43, Iss.5, pp. $51-58$

[12] Intanagonwiwat, C., Govindan,R., Estrin, D.2000. Directed diffusion: A scalable and robust communication paradigm for sensor networks, Proceedings of the Sixth Annual International Conference on Mobile Computing and Networking, ACM Press, pp.56-67

[13] Maroti,M.2004. The flooding time synchronization protocol,Proceedings of the 2 nd international conference on Embedded networked sensor systems

[14] Christos, K. 2009. Time Synchronization in Wireless Sensor Network, Research Academic Computer technology institute, University of Patras

[15] Sichitiu, M.L., and Veerarittiphan,C.2003.Simple, Accurate Time Synchronization for Wireless Sensor Networks, Proc. of International Conference on Wireless Networks (ICWN), pp. 300-305

[16] Gelyan,S.N., Eghbali, AN., Roustapoor,L.2007. SLTP: Scalable Lightweight Time Synchronization Protocol for Wireless Sensor Network, Springer-Verlag Berlin Heidelberg, pp. 536-547

[17] Tanvir,S., Jabeen, F., Iqbal,M. 2012.On Propagation Properties of Beacon Based Localization Protocol for Wireless Sensor Networks, Middle-East Journal of Scientific Research, Vol. 12, No. 2

[18] Zhang,F.,Deng,G.Y.2005.ProbabilisticTimeSynchronizat ion in Wireless Sensor Networks,IEEE International Conference on Wireless Communication, Networking, \& Mobile Computing, Vol.2, pp.980-984
[19] Sivrikaya, F., Yener,B.2004. Time Synchronization in Sensor Networks: A Survey, IEEE Network, Vol.18, Iss.4, pp. 45-50

[20] Keller,M.,Beutel, J., Meier,A., Lim,R., Thiele,L.2009. "Learning from sensor network data". Proceedings of the 7th ACM Conference on Embedded Networked Sensor Systems, New York, NY, USA, pp. 383-384

[21] Shtossel,A., Yaakoby,H., Daliot,A.2009. A Wireless Sensor Network for Rough-terrain Habitat Monitoring, Retreived from leibniz.cs.huji.ac.il/tr/920.pdf

[22] Simon,G.2012.Efficient time-synchronization in ringtopology wireless sensor networks, IEEE International Instrumentation \& Measurement technology Conference, pp.958-962, ISSN 1091-5281

[23] Yoon,S., Sichitiu, M.L.2005. Analysis and Performance Evaluation of a Time Synchronization Protocol for Wireless Sensor Networks, Proc. of The International Conference on Telecommunication Systems, Modeling and Analysis(ICTSM 2005), Dallas, TX,

[24] Albu,R., Labit,Y., Gayraud,T., Berthou,P.2010.An Energy-efficient Clock Synchronization Protocol for Wireless Sensor Networks, Wireless days, IEEE, doi: 10.1109/WD.2010.5657710

[25] Shahzad, K., Ali, A., Gohar, N. D.2008. ETSP: An Energy-efficient Time Synchronization Protocol for Wireless Sensor Networks,IEEE International Conference on Advanced Information Networking \& Application, pp.971-976, ISBN 978-0-7695-3096-3

[26] Sallai,J., Kusy, B., Ledeczi,A., and Dutta,P.2006. On the Scalability of Routing Integrated Time Synchronization, 3rd European Workshop on Wireless Sensor Networks (EWSN). Zürich, Switzerland

[27] Zhou,D., Lai, T.H.2007. A Scalable and Adaptive Clock Synchronization Protocol for IEEE 802.11-Based Multihop Ad Hoc Networks, IEEE International Conference on Mobile Adhoc \& Sensor Conference, ISBN 0-7803-9465-8

[28] Meier,M.2010. Precise Time Synchronization for Wireless Sensor Networks using the Global Positioning System, Semester Thesis of Distributed Computing Group, ETH

[29] Deng,J., Han, R., Mishra,S.2004. Secure and Robust loose time synchronization mechanism for Wireless Sensor Networks, 13th USENIX Security Symposium, San Diego, CA

[30] Elson,J., Romer,K.2002. Wireless Sensor Networks: A New Regime for Time Synchronization, Proceedings of the First Workshop on Hot Topics in Networks, pp.28-29

[31] Kim,R.,Nagayama, T., Jo, H., Spencer, B. F.2012. Preliminary study of low-cost GPS receivers for time synchronization of wireless sensor networks, SPIESmart Structures and Nondestructive Evaluation and Health Monitoring, San Diego, CA, USA

[32] Chen,J., Yu, Q., Zhang,Y., Chen, H.-H., Sun, Y.2010. Feedback-based clock synchronization in wireless sensor networks: a control theoretic approach, IEEE Transactions on Vehicular Technology, Vol. 59, No. 6 , pp. 2963-2973 
[33] Suhonen, J., Kohvakka, M.2012. Low-Power Wireless Sensor Networks: Protocols, Services and Applications, Springer, - Technology \& Engineering, ISSN 2191-8112, pp. 96

[34] Irschik,H., Krommer, M., Watanabe, K., Furukawa, T.2009. Mechanics and Model-Based Control of Smart Materials and Structures, Springer, Technology \& Engineering, pp. 239, ISBN 978-3-211-99484-9

[35] Serpedin,E., Chaudhari, Q. M.2009. Synchronization in Wireless Sensor Networks: Parameter Estimation, Performance Benchmarks, and Protocols, Cambridge University Press, 1st Edition, ISBN-10: 0521764424, $\mathrm{pp} / 232$

[36] Cristian,F.1989. Probabilistic clock synchronization, Distributed Computing, Springer Verilag, Vol.3, pp.146158

[37] Kunz,G., Stoffersz, M., Grossz, J., Wehrle, K.2012. Know Thy Simulation Model: Analyzing Event Interactions for Probabilistic Synchronization in Parallel Simulations, Proceedings of $5^{\text {th }}$ International ICST Conference on Simulation Tool\& Techniques, pp.119128, ISBN: 978-1-4503-1510-4

[38] Sugihara,R.,Gupta,R.K.2011. Clock Synchronization with Deterministic Accuracy Guarantee, Springer, pp.130-146

[39] Ren,F.,Lin, C.2008. Self-correcting time synchronization using reference broadcast in wireless sensor network, IEEE Wireless Communication, ISSN 1536-1284, pp.7985

[40] Gonçalves,F., Suresh, L., Pereira, R. L., Trindade, J., Vazao,T.2013. Light-Weight Time Synchronization For Wireless Sensor Networks, IEEE-Conference on Future Internet Communication, pp.1-8

[41] Wang,N., He, H., Liu, D.2013. A Fault-Tolerant Schema for Clock Synchronization and data aggregation in WSN, International Journal of Networked and Distributed Computing, Vol. 1, No. 1, 46-52

[42] Huang,P-H., Desai, M., Qiu, X., Krishnamachari, B.2009.On the Multi-Hop Performance of SenderReceiver Synchronization Mechanisms", IEEE Transactions on Computers, Vol.58,No.5, ISSN 00189340, pp.577-590

[43] Bholane,S., and Thakore, D.2012. Simulation and Analysis of Receiver-Receiver Time Synchronization in Wireless Sensor Networks, International Journal on Computer Technology \& Applications, Vol 3, Iss.4, pp.1320-1326

[44] Jain, N.,Jain, N.2013. Study of Energy Efficient Time Synchronization Algorithm for the development of Smart Wireless Sensor Network International Journal of Soft Computing and Engineering, ISSN: 2231-2307, Vol.2, Iss.6

[45] Elson,J., Girod, L., Estrin,D.2002. Fine-grained network time synchronization using reference broadcasts, Proceedings of the 5th symposium on Operating systems design and implementation, ACM, Vol.36

[46] Bagul,D., Kurumbanshi, S., Verma, U.2013. Survey on Clock Synchronization in WSN, International Journal of Engineering Science Invention, Vol.2, Iss.12, pp.24-31
[47] Gu,Y., Hwang, J., He, T. , Chang, D. H.2007. uSense: A Unified Asymmetric Sensing Coverage Architecture for Wireless Sensor Networks,IEEE International Conference on Distributed Computing Systems, ISSN 1063-6927, pp.8

[48] Qun, L., Daniela,R.2004. Global clock synchronization in sensor networks, IEEE Transactions on Computers, ISSN 0018-9340, pp. $214-226$

[49] Ram,S.K., Srivastava,M.B. 2003. Timing-sync protocol for sensor networks, First ACM Conference on Embedded Networked SensorSystems, SenSys, pp.138149, ISBN:1-58113-707-9

[50] Mihail,L.S., Veerarittiphan,C.2003.Simple, accurate time synchronization for wireless sensor networks, IEEE Wireless Communications and Networking Conference (WCNC), ISSN 1525-3511, vol.2, pp.1266-1273

[51] Akyildiz, I.W.S.2005. Time-Diffusion Synchronization Protocols for Sensor Networks, IEEE Transactions on Networking, ISSN 1063-6692, pp. $384-397$

[52] Nema,S., Shukla, N.2013. A review on Coverage factors in Wireless Sensor Networks", International Journal of Advanced Research in Computer Engineering \& Technology, Vol.2, Iss.12

[53] Hefeeda,M., Ahmadi, H.2007. A Probabilistic Coverage Protocol for Wireless Sensor Networks, IEEE International Conference on Network Protocols, ISBN 978-1-4244-1588-5, pp.41-50

[54] Jain,N.2013. Study of Energy Efficient Time Synchronization Algorithm for the development of Smart Wireless Sensor Network, International Journal of Soft Computing and Engineering, ISSN: 2231-2307, Vol.2, Iss.6

[55] Ismail,M.M., Sivrikaya,F., Yener, B.2007. Joint Problem of Power Optimal Connectivity and Coverage in Wireless Sensor Networks, Springer, Wireless Networks, Vol.13, Iss.4, pp 537-550

[56] Liu,C., Wu, K., Xiao, Y., Sun, B.2005. Random Coverage with Guaranteed Connectivity: Joint Scheduling for Wireless Sensor Networks, IEEE transactions on parallel and distributed systems, ISSN 1045-9219, pp.562-575

[57] Zhang, H., Hou, J.C.2005. Maintaining Sensing Coverage and Connectivity in Large Sensor Networks, Handbook on Theoretical and Algorithmic Aspects of Sensor, Ad Hoc Wireless, and Peer-to-Peer Networks, ISBN: 978-0-8493-2832-9

[58] Hui,J., Culler,D.2004. The Dynamic Behavior of a Data Dissemination Protocol for Network Programming at Scale, in SenSys,

[59] Fedor,S., Collier, M., Sreenan, C. J.2011. Cross-layer routing and time synchronisation in Wireless Sensor Networks, International Journal Signal and Imaging Systems Engineering, Vol.10, No.3, pp.143 - 159

[60] Varagnolo, D., Chen, P., Schenato, L., Sastry, S.S.2008. Performance analysis of different routing protocols in WSN for real-time estimation, IEEE Conference on Decision and Control, ISSN 0191-2216, ISBN 978-14244-3124-3, pp. 4904 - 4909 
[61] Pister, K.S.J., Doherty, L.2008. TSMP: Time Synchronized Mesh Protocol, Proceedings of IASTED International Symposium Distributed Sensors networks

[62] Ramya,M., Poornima, A.S.2013. Mobile Data Collector For Secure Time Synchronization In Clustered Wireless Sensor Network, An International Journal, ISSN 2320 6632, Vol.1, Iss.2

[63] Choi,J.Y., Lee,W., Lee,K., Choi,S., Kwon,W.H.2006. Aggregation Time Control Algorithm for Time constrained Data Delivery in Wireless Sensor Networks, IEEE vehicular Technology Conference, ISSN 15502252, ISBN 0-7803-9392-9, pp.563-567

[64] Holczer, T., Buttyan,L.2011. Anonymous Aggregator Election and Data Aggregation in Wireless Sensor Networks, Hindawi Publishing Corporation, International
Journal of Distributed Sensor Networks, Article ID 828414, pp.19

[65] Song,H., Zhu, S., Cao, G.2005. Attack-Resilient Time Synchronization for Wireless Sensor Networks, IEEE International Conference on Mobile Adhoc and Sensor System Conference, ISBN 0-7803-9465-8, Vol.8

[66] Solis, I., Obraczka, K.2004. The Impact of Timing in Data Aggregation for Sensor Networks, IEEE International Conference on Communication, ISBN 07803-8533-0, Vol.6, pp.3640-3645

[67] Krishnamachari,B., Estrin,D., and Wicker, S.2002. Impact of data aggregation in wireless sensor networks, International Workshop on DIstributed Event-Based Systems, Vienna, Austria. 